Research Article

\title{
Research on Error Estimations of the Interpolating Boundary Element Free-Method for Two-Dimensional Potential Problems
}

\author{
Jufeng Wang, ${ }^{1}$ Fengxin Sun $\mathbb{D}^{2},{ }^{2}$ and Ying $\mathrm{Xu} \mathbb{D}^{1}$ \\ ${ }^{1}$ College of Finance \& Information, Ningbo University of Finance \& Economics, Ningbo 315175, China \\ ${ }^{2}$ Faculty of Science, Ningbo University of Technology, Ningbo 315016, China
}

Correspondence should be addressed to Fengxin Sun; fengxin@nbut.edu.cn

Received 15 June 2020; Accepted 29 June 2020; Published 25 July 2020

Guest Editor: Miaojuan Peng

Copyright (C) 2020 Jufeng Wang et al. This is an open access article distributed under the Creative Commons Attribution License, which permits unrestricted use, distribution, and reproduction in any medium, provided the original work is properly cited.

The interpolating boundary element-free method (IBEFM) is a direct solution method of the meshless boundary integral equation method, which has high efficiency and accuracy. The IBEFM is developed based on the interpolating moving least-squares (IMLS) method and the boundary integral equation method. Since the shape function of the IMLS method satisfies the interpolation characteristics, the IBEFM can directly and accurately impose the essential boundary conditions, which overcomes the shortcomings of the original boundary element-free method in enforcing the essential boundary approximately. This paper will study the error estimations of the IBEFM for two-dimensional potential problems and the relationship between the errors and the influence radius and the condition number of the coefficient matrix. Two numerical examples are presented to verify the correctness of the theoretical results in this paper.

\section{Introduction}

The meshless method is an important numerical method after the finite element method, which has been widely used in scientific engineering calculation [1-5]. Based on different methods of constructing the shape function and problem discretization, scholars have proposed a series of meshless methods, such as the smoothed particle hydrodynamics $(\mathrm{SPH})$ method $[6,7]$, element-free Galerkin (EFG) method [8], the singular boundary method (SBM) [9], radial basis functions $[10,11]$, boundary knot method (BKM) [12], meshless local Petrov-Galerkin (MLPG) method [13], complex variable element-free Galerkin (ICVFG) method $[14,15]$, point interpolation method (PIM) [16], method of fundamental solutions [17], and dimension splitting meshless method [18].

The traditional least-squares method (LSM) is a commonly used method in numerical computation, which obtains the optimal solution by seeking the minimum deviation of all data $[19,20]$. When studying surface fitting, based on the LSM, Lancaster and Salkauskas proposed the moving least-squares (MLS) method [21], which is one of the most important methods to construct the trial function in the meshless method. The meshless method based on the MLS method has high numerical accuracy [22, 23]. However, the shape function of the MLS method does not satisfy the property of the Kronecker $\delta$ function [24], which increases the difficulty of dealing with the boundary conditions, and also raises a lot of extra computational burden. The meshless method of the MLS method class cannot apply essential boundary conditions directly and simply as the finite element method [25-27]. To solve this problem, by using the singular weight function, Lancaster and Salkauskas proposed the interpolation moving least square (IMLS) method [21], in which the shape function meets the interpolation characteristics at the nodes. The meshless method based on the IMLS method can directly and simply impose the essential boundary conditions [28], which improve calculation accuracy and efficiency. Based on the IMLS method, different meshless methods are proposed to solve various problems [29-33].

Based on the MLS method and boundary integral equation method, the boundary element-free method (BEFM) was developed by Cheng and Peng [34]. The BEFM 
is a meshless boundary integral equation method. Compared with the boundary point method, the boundary integral equation adopted in BEFM is a common and more regular form, and there is need neither to set Gauss points in the neighbourhood of the source point nor to choose the so-called "evaluation point," so the solution process of the BEFM is more straightforward. The BEFM uses the real solutions of the node variables as the unknown quantities, while the boundary point method and the local boundary integral equation method both use the approximate solutions of the node variables as the unknown, so the BEFM is a direct numerical mesh-free method of the boundary integral equation and has greater computational precision [35-38].

In the BEFM, the essential boundary conditions are directly imposed. However, because the shape function of the MLS method does not satisfy the interpolation characteristics at the nodes, the essential boundary conditions are only approximately satisfied, which may lead to reduction in calculation accuracy. In order to improve this deficiency, by taking into account the interpolation characteristics of the IMLS method, Ren et al. also proposed the interpolating boundary element-free method (IBEFM) [39], which has higher calculation accuracy than the BEFM [40, 41]. By using the improved IMLS method with a nonsingular weight function, Wang et al. also developed an enhanced IBEFM for the potential problems [42]. As a direct numerical mesh-free method, the IBEFM has both the advantages of high accuracy and the ability to directly and accurately apply boundary conditions.

The BEFM has been widely used in various fields $[37,39,43]$. However, there is still little research on the error estimation of the BEFM. Based on the error estimation of the IMLS method and related matrix theory, this paper will study the error estimation of the IBEFM for the two-dimensional potential problems. The relationship between the errors and the influence radius and the condition number of the coefficient matrix is studied, and some numerical examples are presented to verify the correctness of the theoretical results.

\section{The IBEFM for the Potential Problems}

Consider the following two-dimensional Poisson equation,

$$
\nabla^{2} u(x)+b(x)=0, \quad x=\left(x_{1}, x_{2}\right) \in \Omega,
$$

with the Dirichlet boundary condition,

$$
u(x)=\bar{u}(x), \quad x \in \Gamma_{u},
$$

and the Neumann boundary condition,

$$
q(x)=\frac{\partial u(x)}{\partial n}=\bar{q}(x), \quad x \in \Gamma_{q},
$$

where $\Omega$ is the problem field, $u(x)$ represents the potential of the field function, $b(x)$ is the given source function, $\Gamma$ is the boundary of $\Omega$ satisfying $\Gamma=\Gamma_{u} \cup \Gamma_{q}, \bar{u}$ denotes the known potential function on the Dirichlet boundary $\Gamma_{u}, \bar{q}$ is the known potential gradient on the Neumann boundary $\Gamma_{q}$, and $n$ is the cosine of the outer normal direction on the boundary $\Gamma$.

The integral equation can be obtained using the weighted residual method:

$$
\begin{aligned}
\int_{\Omega}\left(\nabla^{2} u(x)-b(x)\right) u^{*}(\xi, x) \mathrm{d} \Omega & =\int_{\Gamma_{q}}(q(x)-\bar{q}(x)) u^{*}(\xi, x) \mathrm{d} \Gamma \\
& -\int_{\Gamma_{u}}((x)-\bar{u}(x)) q^{*}(\xi, x) \mathrm{d} \Gamma,
\end{aligned}
$$

where $x$ and $\xi$ represent, respectively, the field point and source point and $u^{*}$ is the basic solution of the Poisson equation (1) satisfying

$$
\nabla^{2} u^{*}=-\delta(\xi, x),
$$

where $\delta(\xi, x)$ is the Dirac $\delta$ function. Then, it follows that

$$
\begin{aligned}
u^{*}(\xi, x) & =\frac{1}{2 \pi} \ln \left(\frac{1}{r(\xi, x)}\right), \\
q^{*}(\xi, x) & =\frac{\partial u^{*}(\xi, x)}{\partial n(x)}, \\
r(\xi, x) & =\sqrt{\left(\xi_{1}-x_{1}\right)^{2}+\left(\xi_{2}-x_{2}\right)^{2}} .
\end{aligned}
$$

Performing the partial integration on (4) and using the properties of the basic solution of the Poisson equation, when the source point $\xi$ is within $\Omega$, the following boundary integral equation can be obtained:

$$
\begin{aligned}
u(\xi)= & \int_{\Gamma} q(x) u^{*}(\xi, x) \mathrm{d} \Gamma-\int_{\Gamma} u(x) q^{*}(\xi, x) \mathrm{d} \Gamma \\
& -\int_{\Omega} b(x) u^{*}(\xi, x) \mathrm{d} \Omega,
\end{aligned}
$$

when the source point $\xi$ is on the boundary $\Gamma$, and the boundary integral equation is

$$
\begin{aligned}
C(\xi) u(\xi)= & \int_{\Gamma} q(x) u^{*}(\xi, x) \mathrm{d} \Gamma-\int_{\Gamma} u(x) q^{*}(\xi, x) \mathrm{d} \Gamma \\
& -\int_{\Omega} b(x) u^{*}(\xi, x) \mathrm{d} \Omega,
\end{aligned}
$$

where $C(\xi)$ is a free term determined by the geometry of the point $\xi$, and

$$
C(\xi)= \begin{cases}\frac{1}{2}, & \text { when } \xi \text { is a smooth boundary point, } \\ \frac{\theta}{2 \pi}, & \text { when } \xi \text { is a boundary corner point, }\end{cases}
$$

where $\theta=\theta_{2}-\theta_{1}$ and according to the boundary integration direction, $\theta_{1}$ and $\theta_{2}$ are the angles between the boundary tangent and the coordinate axis away from the corner point and near the corner point, respectively.

For the two-dimensional potential problem, the points on the boundary can be seen as points in one-dimensional space. $\forall x \in \Gamma$ can be expressed by curvilinear coordinates $s$, that is $x=x(s)$. In this article, the curvilinear coordinates 
are taken as the arc length of the curve. Let $\mathbf{X}=\left\{x_{1}, x_{2}, \ldots, x_{N}\right\}$ denote the coordinate set of all nodes on the boundary, where $N$ represents the total number of nodes. Suppose the arc length coordinate of $x_{I}$ to be $s_{I}$, and this node is also called a node $s_{I}$. The union of the influence domains of all nodes must cover the entire boundary, and for $x_{I} \in \Gamma$, it is assumed that the influence domain $R\left(x_{I}\right)$ is

$$
R\left(x_{I}\right):=\left\{x(s) \in \Gamma:\left|s-s_{I}\right| \leq \rho_{I}\right\},
$$

where $\rho_{I}$ is the influence radius of node $x_{I}$.

Suppose $\left\{s_{1}, s_{2}, \ldots, s_{n_{I}}\right\}$ are the nodes with the influence domain covering the $x(s)$. Then, from the IMLS method, the approximation functions can be obtained as follows:

$$
\begin{aligned}
& u(x) \approx u^{h}(x(s))=\sum_{I=1}^{n_{I}} \Phi_{I}(s) u_{I}, \\
& q(x) \approx q^{h}(x(s))=\sum_{I=1}^{n_{I}} \Phi_{I}(s) q_{I},
\end{aligned}
$$

where $u_{I}=u\left(x_{I}\right)$ and $q_{I}=q\left(x_{I}\right)$.

Substituting (13) and (14) into (10), it follows that

$$
\begin{aligned}
C\left(x_{I}\right) u\left(x_{I}\right)= & \int_{\Gamma} u^{*}\left(x_{I}, x\right) \sum_{J=1}^{n_{I}} \Phi_{J}(s) q_{J} \mathrm{~d} \Gamma \\
& -\int_{\Gamma} q^{*}\left(x_{I}, x\right) \sum_{J=1}^{n_{I}} \Phi_{J}(s) u_{J} \mathrm{~d} \Gamma \\
& -\int_{\Omega} b(x) u^{*}\left(x_{I}, x\right) \mathrm{d} \Omega .
\end{aligned}
$$

To calculate the definite integral, the boundary $\Gamma$ is divided into some integral subdomains $\Gamma_{n}, n=1,2, \ldots, N_{e}$. $N_{e}$ is the total number of boundary integral subdomains, and only one point of any adjacent subdomains is connected. These integral subdomains are independent of the nodes distribution on the boundary, and they are only used to calculate the numerical integral.

To calculate $\int_{\Omega} b(x) u^{*}\left(x_{I}, x\right) \mathrm{d} \Omega$, the field $\Omega$ is divided into $N_{c}$ integral subfields. $A_{c}$ is the area of the subfield $c$, and $K$ is the number of Gauss integral points in the integral subfield $c . w_{k}$ is the integral weight coefficient corresponding to the $k$-th Gauss integral point, and $\left(b u^{*}\right)_{k}$ is the value of $b u^{*}$. Therefore, equation (15) can be written as

$$
\begin{aligned}
C\left(x_{I}\right) u\left(x_{I}\right)= & \sum_{n=1}^{N_{e}} \int_{\Gamma_{n}} u^{*}\left(x_{I}, x\right) \sum_{J=1}^{n_{I}} \Phi_{J}(s) q_{J} \mathrm{~d} \Gamma \\
& -\sum_{n=1}^{N_{e}} \int_{\Gamma_{n}} q^{*}\left(x_{I}, x\right) \sum_{J=1}^{n_{I}} \Phi_{J}(s) u_{J} \mathrm{~d} \Gamma \\
& -\sum_{c=1}^{N_{c}}\left(\sum_{k=1}^{K} w_{k}\left(b u^{*}\right)_{k}\right) A_{c} .
\end{aligned}
$$

By substituting each node on the boundary into (16) and combining the equations, the following equation can be obtained:

$$
\mathbf{B}+\mathbf{H U}=\mathbf{G Q},
$$

where $\mathbf{H}=\left(H_{I J}\right)_{N \times N}, \mathbf{G}=\left(G_{I J}\right)_{N \times N}, \mathbf{B}=\left(B_{I}\right)_{N \times 1}$, and

$$
\begin{aligned}
H_{I J} & =\sum_{n=1}^{N_{e}} \int_{\Gamma_{n}} q^{*}\left(x_{I}, x\right) \Phi_{J}(s) \mathrm{d} \Gamma+\delta_{I J} C\left(x_{I}\right), \\
G_{I J} & =\sum_{n=1}^{N_{e}} \int_{\Gamma_{n}} u^{*}\left(x_{I}, x\right) \Phi_{J}(s) \mathrm{d} \Gamma \\
B_{I} & =\sum_{c=1}^{N_{c}}\left(\sum_{k=1}^{K} w_{k}\left(b(x) u^{*}\left(x_{I}, x\right)\right)_{k}\right) A_{c}, \\
\mathbf{U} & =\left(u_{1}, u_{2}, \ldots, u_{N}\right)^{T} \\
\mathbf{Q} & =\left(q_{1}, q_{2}, \ldots, q_{N}\right)^{T} .
\end{aligned}
$$

In the calculation of the boundary integral equation (16), in most cases, the boundary source point $x_{I}$ is not on the integral subdomain $\Gamma_{n}$, and the integration has no singularity. The Gauss integration method can be used directly. If the source point coincides with the integration point, the basic solution will lead to the integration singularity. At this time, the Gauss integration point can be rearranged to eliminate the singularity.

Because the shape function of the IMLS method satisfies the property of the Kronecker $\delta$ function, the boundary condition can be easily and directly applied. Substituting the potential and potential gradient at the nodes on the boundary into (17), the potential and potential gradient at all the nodes on the boundary can be solved. Then, from (9), the potential at $\forall \xi \in \Omega$ can be obtained as

$$
\begin{aligned}
u(\xi)= & \sum_{n=1}^{N_{e}} \int_{\Gamma_{n}} u^{*}(\xi, x) \sum_{I=1}^{n_{I}} \Phi_{I}(s) q_{I} \mathrm{~d} \Gamma \\
& -\sum_{n=1}^{N_{e}} \int_{\Gamma_{n}} q^{*}(\xi, x) \sum_{I=1}^{n_{I}} \Phi_{I}(s) u_{I} \mathrm{~d} \Gamma \\
& -\sum_{c=1}^{N_{c}}\left(\sum_{k=1}^{K} w_{k}\left(b u^{*}\right)_{k}\right) A_{c} .
\end{aligned}
$$

Calculating differential for (19), the potential gradient of the interior point can be obtained:

$$
\begin{aligned}
u_{, i}(\xi)= & \sum_{n=1}^{N_{e}} \int_{\Gamma_{n}} u_{, i}^{*}(\xi, x) \sum_{I=1}^{n_{I}} \Phi_{I}(s) q_{I} \mathrm{~d} \Gamma \\
& -\sum_{c=1}^{N_{c}} \int_{\Gamma_{n}} q_{, i}^{*}(\xi, x) \sum_{I=1}^{n_{I}} \Phi_{I}(s) q_{I} \mathrm{~d} \Gamma \\
& -\sum_{c=1}^{N_{c}}\left(\sum_{k=1}^{K} w_{k}\left(b u_{, i}^{*}\right)_{k}\right) A_{c}, \quad i=1,2 .
\end{aligned}
$$

The abovementioned equation is the IBEFM for twodimensional potential problems. 


\section{Error Estimations of the IBEFM for Two- Dimensional Potential Problems}

For simplicity, let $E_{1}=\left\{x_{1}, x_{2}, \ldots, x_{N_{1}}\right\}$ be the set of all nodes on the Dirichlet boundary, and $E_{2}=\left\{x_{N_{1}+1}\right.$, $\left.x_{N_{1}+2}, \ldots, x_{N}\right\}$ be the set of all nodes on the Neumann boundary. For $\forall x_{I} \in X$, assume that there is a constant $c_{I}$ such that $\rho \leq c_{I} \rho_{I}$, and the basis functions used in the IMLS method are complete polynomials of order $m$.

Since there is no intersection between $\Gamma_{u}$ and $\Gamma_{q}$, then in (17), when the $i$-th element in $\mathbf{U}$ is unknown, the $i$-th element in $\mathbf{Q}$ must be known; otherwise, when the $i$-th element in $\mathbf{U}$ is known, the $i$-th element in $\mathbf{Q}$ must be unknown. By shifting the term, all the unknown quantities are moved to the left end, and the known quantities are moved to the right end. Suppose the unknown quantities to be solved at the right end be $\mathbf{X}$, and its coefficient matrix be $\mathbf{A}$, and at the right end, the known column vector is denoted by $\mathbf{f}$ after the combining operation. Then, (17) can be transformed into the following linear equation:

$$
\mathbf{A X}=\mathbf{f}
$$

where $\mathbf{A}$ is an $N \times N$ matrix and $\mathbf{X}$ and $\mathbf{f}$ are $N \times 1$ column vectors.

Suppose $\widehat{U}=\left(\widehat{u}_{1}, \widehat{u}_{2}, \ldots, \widehat{u}_{N}\right)^{T}$ and $\widehat{Q}=\left(\widehat{q}_{1}, \widehat{q}_{2}, \ldots, \widehat{q}_{N}\right)^{T}$ are the numerical solutions of the potential and potential gradient obtained by the IBEFM. Since the IMLS method satisfies interpolation characteristics, it follows on the Dirichlet and Neumann boundaries that

$$
\begin{array}{ll}
\widehat{u}_{I}=\bar{u}\left(x_{I}\right), & I=1,2, \ldots, N_{1}, \\
\widehat{q}_{I}=\bar{q}\left(x_{I}\right), & I=N_{1}+1, N_{1}+2, \ldots, N .
\end{array}
$$

Let $\widehat{X}=\left(\widehat{q}_{1}, \ldots, \widehat{q}_{N_{1}}, \widehat{u}_{N_{1}+1}, \ldots, \widehat{u}_{N}\right)^{T}$ be the solution of the linear system (21), that is,

$$
\mathbf{A} \widehat{X}=\mathbf{f} .
$$

Let $u(x(s))$ and $q(x(s))$ be the exact solutions to the potential and potential gradient, respectively. Also, let

$$
\mathbf{X}^{e}=\left(q\left(x_{1}\right), \ldots, q\left(x_{N_{1}}\right), u\left(x_{N_{1}+1}\right), \ldots, u\left(x_{N}\right)\right)^{T} .
$$

Therefore, at the boundary node, the following theorem exists between the exact solution and the numerical solution of the IBEFM.

Theorem 1. When the boundary is sufficiently smooth and the radius of the influence domain of the boundary node is small enough, there exists a constant $C$ independent with $\rho$ such that

$$
\left\|\mathbf{X}^{e}-\widehat{X}\right\|_{\infty} \leq C k(u, q) \frac{\operatorname{cond}(\mathbf{A})}{\|\mathbf{f}\|_{\infty}} \rho^{m+1},
$$

where $\|\cdot\|_{\infty}$ represents the maximum norm of a vector (matrix) and

$$
k(u, q)=\max \left\{\|q\|_{\Gamma, m+1},\|u\|_{\Gamma, m+1}\right\} \max \left\{\|q\|_{\Gamma, \infty},\|u\|_{\Gamma, \infty}\right\} .
$$

Proof. Let

$$
\begin{aligned}
& u(x(s))=u^{h}(x(s))+r_{1}(s), \\
& q(x(s))=q^{h}(x(s))+r_{2}(s),
\end{aligned}
$$

where $u^{h}(x(s))$ and $q^{h}(x(s))$ are the approximation functions obtained by the IMLS method.

Then, from the error estimates of the IMLS method $[28,44,45]$, there exist

$$
\begin{gathered}
\left\|r_{1}(s)\right\|_{\Gamma, 0} \leq C \rho^{m+1}\|u\|_{\Gamma, m+1}, \\
\left\|r_{2}(s)\right\|_{\Gamma, 0} \leq C \rho^{m+1}\|q\|_{\Gamma, m+1} .
\end{gathered}
$$

It follows from (10) that

$$
\begin{aligned}
C(\xi) u(\xi)= & \int_{\Gamma}\left[q^{h}(x)+r_{2}(s)\right] u^{*}(\xi, x) \mathrm{d} \Gamma \\
& -\int_{\Gamma}\left[u^{h}(x)+r_{1}(s)\right] q^{*}(\xi, x) \mathrm{d} \Gamma \\
& -\int_{\Omega} b(x) u^{*}(\xi, x) \mathrm{d} \Omega,
\end{aligned}
$$

where $\xi$ is the source point on the boundary $\Gamma$.

Then,

$$
\begin{aligned}
C\left(\xi_{I}\right) u\left(\xi_{I}\right)= & \int_{\Gamma}\left[\sum_{J=1}^{n_{I}} \Phi_{J}(s) q\left(x_{J}\right)+r_{2}(s)\right] u^{*}\left(\xi_{I}, x\right) \mathrm{d} \Gamma \\
& -\int_{\Gamma}\left[\sum_{J=1}^{n_{I}} \Phi_{J}(s) u\left(x_{J}\right)+r_{1}(s)\right] q^{*}\left(\xi_{I}, x\right) \mathrm{d} \Gamma \\
& -\int_{\Omega} b(x) u^{*}\left(\xi_{I}, x\right) \mathrm{d} \Omega
\end{aligned}
$$

where $\xi_{I}$ is a boundary node.

The same numerical integration is used for (16) and (31). Also, using the interpolation properties of the IMLS method, we have

$$
\mathbf{B}+\mathbf{H U}^{e}=\mathbf{G Q}^{e}+\boldsymbol{\varepsilon},
$$

where

$$
\begin{aligned}
\mathbf{U}^{e} & =\left(u\left(x_{1}\right), u\left(x_{2}\right), \ldots, u\left(x_{N}\right)\right)^{T}, \\
\mathbf{Q}^{e} & =\left(q\left(x_{1}\right), q\left(x_{2}\right), \ldots, q\left(x_{N}\right)\right)^{T},
\end{aligned}
$$

and $\varepsilon$ is an $N \times 1$ column vector with the element given by

$$
\varepsilon_{I}=\int_{\Gamma} r_{2}(s) u^{*}\left(\xi_{I}, x\right) \mathrm{d} \Gamma-\int_{\Gamma} r_{1}(s) q^{*}\left(\xi_{I}, x\right) \mathrm{d} \Gamma .
$$

By adopting the same shifting arrangement of (21) for (32), there exists

$$
\mathbf{A X}^{e}=\mathbf{f}+\boldsymbol{\varepsilon}
$$

where 


$$
\mathbf{X}^{e}=\left(q\left(x_{1}\right), \ldots, q\left(x_{N_{1}}\right), u\left(x_{N_{1}+1}\right), \ldots, u\left(x_{N}\right)\right)^{T} .
$$
that

According to matrix theory, it follows from (23) and (35)

$$
\left\|\mathbf{X}^{e}-\widehat{X}\right\|_{\infty} \leq \operatorname{cond}(\mathbf{A}) \frac{\left\|\mathbf{X}^{e}\right\|_{\infty} \cdot\|\varepsilon\|_{\infty}}{\|\mathbf{f}+\varepsilon\|_{\infty}},
$$

where $\|\cdot\|_{\infty}$ represents the maximum norm of the vector, e.g., $\| \varepsilon_{\infty}=\max _{I}\left|\varepsilon_{I}\right|$.

From (28), (29), and (34), we have

$$
\begin{aligned}
\left|\varepsilon_{I}\right| \leq & {\left[\int_{\Gamma}\left(r_{2}(s)\right)^{2} \mathrm{~d} \Gamma\right]^{1 / 2}\left[\int_{\Gamma}\left(u^{*}\left(\xi_{I}, x\right)\right)^{2} \mathrm{~d} \Gamma\right]^{1 / 2} } \\
& +\left[\int_{\Gamma}\left(r_{1}(s)\right)^{2} \mathrm{~d} \Gamma\right]^{1 / 2}\left[\int_{\Gamma}\left(q^{*}\left(\xi_{I}, x\right)\right)^{2} \mathrm{~d} \Gamma\right]^{1 / 2} \\
& \leq C \max \left\{\|q\|_{\Gamma, m+1},\|u\|_{\Gamma, m+1}\right\} \rho^{m+1} .
\end{aligned}
$$

Then,

$$
\left\|\mathbf{X}^{e}-\hat{X}\right\|_{\infty} \leq C k(u, q) \frac{\operatorname{cond}(\mathbf{A})}{\|\mathbf{f}+\boldsymbol{\varepsilon}\|_{\infty}} \rho^{m+1},
$$

where

$$
k(u, q)=\max \left\{\|q\|_{\Gamma, m+1},\|u\|_{\Gamma, m+1}\right\} \max \left\{\|q\|_{\Gamma, \infty},\|u\|_{\Gamma, \infty}\right\} .
$$

When the Gaussian point and the source point do not coincide, it can be seen from equations (6) and (7) that the basic solutions are bounded. Also, from the errors of the IMLS method [28, 45], there exists constant $C$ independent of $\rho$ such that

$$
\left|\Phi_{J}(s)\right| \leq C .
$$

Then, on the boundary, when $q_{J}$ or $u_{J}$ is given, the expression $u^{*}\left(x_{I}, x\right) \sum_{I=1}^{n_{I}} \Phi_{I}(s) q_{J}$ or $q^{*}\left(x_{I}, x\right) \sum_{I=1}^{n_{I}} \Phi_{I}(s) u_{I}$ must be bounded. Thus, it can be seen from (34)-(36) that the element of the vector $\mathbf{f}$ must be a bounded value that is not a constant equal to 0 . From (38), when the radius of the influence domain is small enough, the element value of $\varepsilon$ is much smaller than that of $\mathbf{f}$, which is almost negligible. Then, the theorem can be directly obtained from (39).

When the error caused by numerical integration is ignored, the following error estimate can be obtained.

Theorem 2. Suppose $u(\xi)$ is the solution of (1) determined by (9) and $\widehat{u}(\xi)$ is the numerical solution of the IBEFM solved from (19). When the boundary is sufficiently smooth and the radius $\rho$ is small enough, there is a constant $C$ independent of $\rho$ such that

$$
|u(\xi)-\widehat{u}(\xi)| \leq C k(u, q) \frac{\operatorname{cond}(\mathbf{A})}{\|\mathbf{f}\|_{\infty}} \rho^{m+1} .
$$

Proof. From (19) and (9), we have

$$
\begin{aligned}
u(\xi)-\widehat{u}(\xi) & =\int_{\Gamma}\left[q(x)-\sum_{J=1}^{n_{I}} \Phi_{J}(s) \widehat{q}_{J}\right] u^{*}(\xi, x) \mathrm{d} \Gamma \\
& -\int_{\Gamma}\left[u(x)-\sum_{J=1}^{n_{I}} \Phi_{J}(s) \widehat{u}_{J}\right] q^{*}(\xi, x) \mathrm{d} \Gamma .
\end{aligned}
$$

Then,

$$
\begin{aligned}
u(\xi)-\widehat{u}(\xi)= & \int_{\Gamma}\left(\left(q(x)-q^{h}(x)\right)+\left(q^{h}(x)-\sum_{J=1}^{n_{I}} \Phi_{J}(s) \widehat{q}_{J}\right)\right) u^{*}(\xi, x) \mathrm{d} \Gamma \\
& -\int_{\Gamma}\left(\left(u(x)-u^{h}(x)\right)+\left(u^{h}(x)-\sum_{J=1}^{n_{I}} \Phi_{J}(s) \widehat{u}_{J}\right)\right) q^{*}(\xi, x) \mathrm{d} \Gamma \\
= & \int_{\Gamma}\left(\left(q(x)-q^{h}(x)\right)+\sum_{J=1}^{n_{I}} \Phi_{J}(s)\left(q_{J}-\widehat{q}_{J}\right)\right) u^{*}(\xi, x) \mathrm{d} \Gamma \\
& -\int_{\Gamma}\left(\left(u(x)-u^{h}(x)\right)+\sum_{J=1}^{n_{I}} \Phi_{J}(s)\left(u_{J}-\widehat{u}_{J}\right)\right) q^{*}(\xi, x) \mathrm{d} \Gamma .
\end{aligned}
$$

From the errors of the IMLS method [28, 45], there exists constant $C$ independent of $\rho$ such that

$$
\left|\Phi_{J}(s)\right| \leq C .
$$

From (25), it follows that

$$
\begin{aligned}
& \left|q_{J}-\widehat{q}_{J}\right| \leq C k(u, q) \frac{\operatorname{cond}(\mathbf{A})}{\|\mathbf{f}\|_{\infty}} \rho^{m+1}, \\
& \left|u_{J}-\widehat{u}_{J}\right| \leq C k(u, q) \frac{\operatorname{cond}(\mathbf{A})}{\|\mathbf{f}\|_{\infty}} \rho^{m+1} .
\end{aligned}
$$

Then, using trigonometric inequality and Hölder's inequality and substituting (28), (29), (46), and (47) into (44), the theorem holds.

The value of $\|\mathbf{f}\|_{\infty}$ contained in Theorem 2 is determined by the value of the potential function and its potential gradient on the boundary. Therefore, when the solution of the problem (1) is sufficiently smooth, there are three main aspects that affect the errors of the numerical solution: the size of the influence domain of nodes, that is, the density of nodes; the order of the complete polynomials basis function of the IMLS method; and the condition number of the coefficient matrix A. 


\section{Numerical Examples}

Two numerical examples will be given to verify the error estimation of the interpolated boundary element-free method in this section. The linear basis function is used in all calculations. The radius of influence domain is $\rho=2 h$, where $h$ is the arc length between two adjacent nodes.

Example 1. Consider the following Laplace equation:

$$
\nabla^{2} T=0, \quad\left(x_{1}, x_{2}\right) \in \Omega
$$

where $\Omega$ is a circular domain with boundary $\Gamma: x_{1}^{2}+x_{2}^{2}=1$. The essential boundary condition is

$$
T\left(x_{1}, x_{2}\right)=\bar{T}, \quad\left(x_{1}, x_{2}\right) \in \Gamma, x_{2} \geq 0,
$$

and the derivative boundary condition is

$$
\frac{\partial}{\partial n} T\left(x_{1}, x_{2}\right)=\bar{q}, \quad\left(x_{1}, x_{2}\right) \in \Gamma, x_{2}<0 .
$$

$\bar{T}$ and $\bar{q}$ are the known functions determined by the following exact solution:

$$
T\left(x_{1}, x_{2}\right)=e^{x_{2}} \sin x_{1} .
$$

When 30 regular nodes are used (as shown in Figure 1), in Figure 2, the exact and numerical results on the inner circle with radii of $0.5,0.7$, and 0.9 are given. From the figure, it can be seen that the numerical solution obtained by the IBEFM has high accuracy. Figure 3 shows the absolute errors of the solutions obtained by the BEFM and IBEFM on the half circle with a radius 0.5 , respectively, in which the BEFM and IBEFM adopt the same boundary integral grid. It can be seen that the solutions of the IBEFM have better accuracy, which also shows that the IBEFM has better advantages than the BEFM. The MATLAB programs of the BEFM and IBEFM run 100 times, respectively, and their average CPU time is $2.2403 \mathrm{~s}$ and $1.9519 \mathrm{~s}$. This shows that the IBEFM has high computational efficiency.

To study the convergence of the IFEFM with respect to the radius $\rho$, when $10,20,30,40$, and 50 regular nodes are used, the corresponding absolute errors between the analytical and numerical solutions at points $(0.7,0.5)$ and $(0.3,0.9)$ are as shown in Figures 4 and 5 , respectively. As can be seen from these figures, the solution of the IBEFM has a good convergence order. It has a better convergence order than the error estimate given by this paper, which also shows the correctness of the theorem.

To study the influence of the condition number of the coefficient matrix on the error, we apply the random function "rand" of MATLAB software to randomly generate 50 kinds of irregular node distribution with 30 nodes. Then, under each irregular node distribution, the IBEFM is used to solve the numerical solutions at internal points $(0.7,0.5)$ and $(0.3,0.9)$.

Figures 6 and 7 show, respectively, the changes of the absolute errors at $(0.7,0.5)$ and $(0.3,0.9)$ for the different matrix condition numbers. As can be seen from the figure, although the error is not strictly worse as the condition number becomes more massive, on the whole, there is a

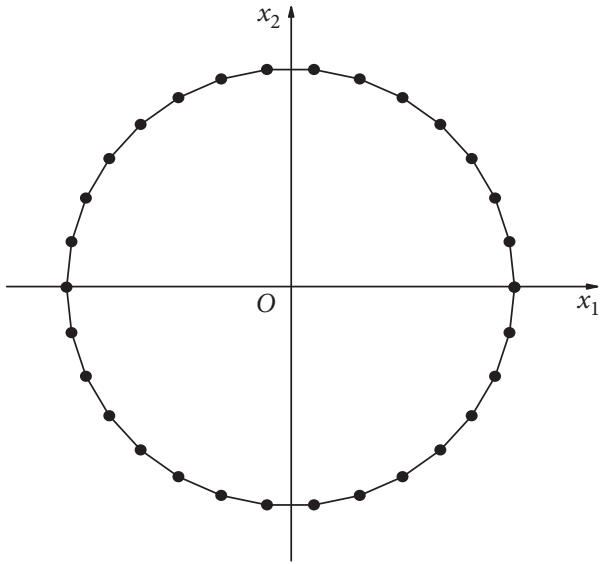

Figure 1: Nodes distribution for Example 1.
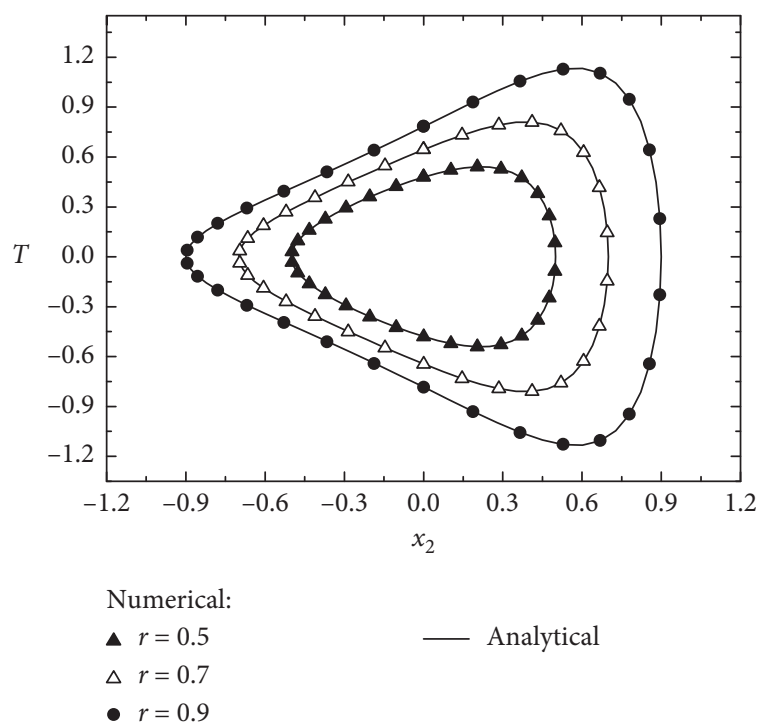

Figure 2: The exact and numerical results on the inner circle with radii of $0.5,0.7$, and 0.9 .

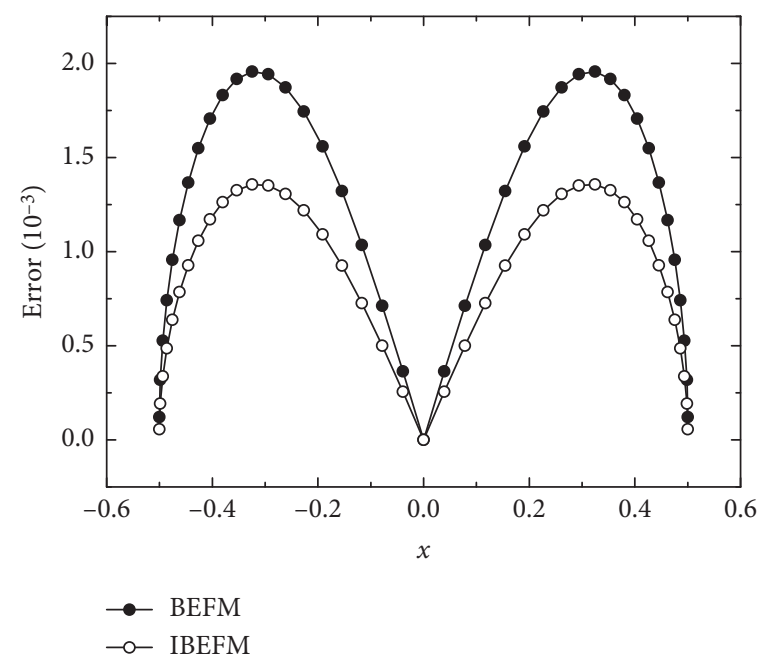

FIgUre 3: The absolute errors of the solutions obtained by the BEFM and IBEFM, respectively, on the half circle with 0.5 radius. 


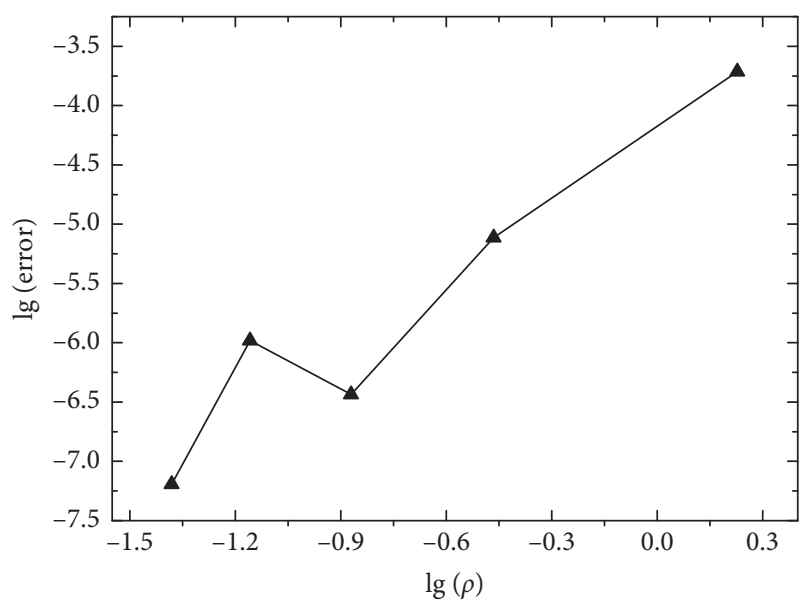

FIGURE 4: The variation of the absolute error for different influence radii at $(0.7,0.5)$.

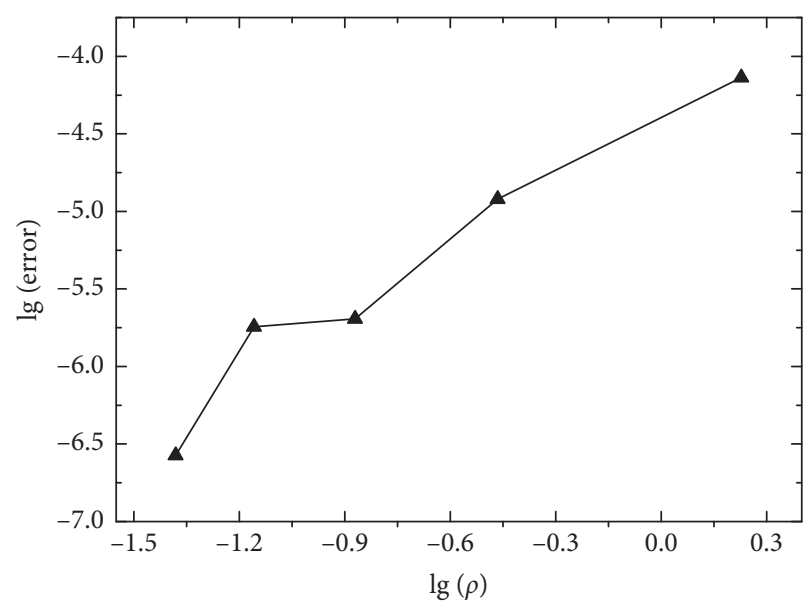

Figure 5: The variation of the absolute error for different influence radii at $(0.3,0.9)$.

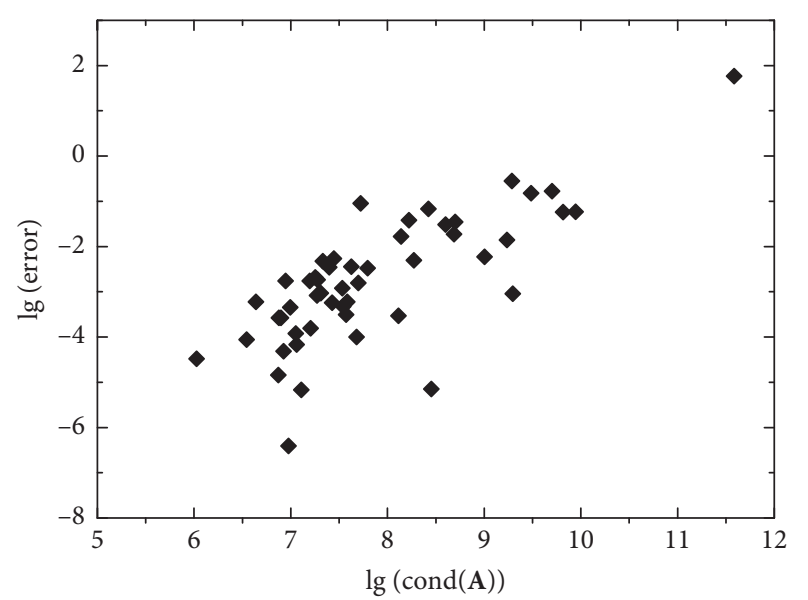

Figure 6: The variation of the absolute error for different cond(A) at $(0.7,0.5)$.

great correlation between the condition number and the error. When the value of the condition number becomes significantly larger, the error will deteriorate. This means

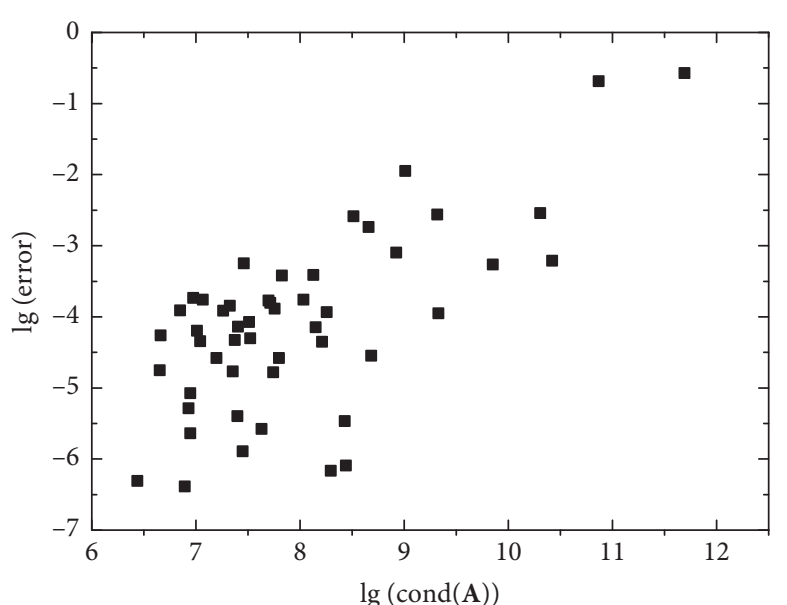

FIgURE 7: The variation of the absolute error for different cond (A) at $(0.3,0.9)$.

that when the condition number of the coefficient matrix becomes considerably larger and the error will become larger, which is consistent with the theoretical results in the paper.

Example 2. Consider the Poisson equation with a square temperature field:

$$
\nabla^{2} T\left(x_{1}, x_{2}\right)=2\left(x_{1}+x_{2}-x_{1}^{2}-x_{2}^{2}\right), \quad 0 \leq x_{1} \leq 1,0 \leq x_{2} \leq 1 .
$$

The Dirichlet and Neumann boundary conditions are, respectively,

$$
\begin{aligned}
T\left(x_{1}, x_{2}\right) & =\bar{T}, \quad\left(x_{1}, x_{2}\right) \in \Gamma, x_{2}=0 \text { or } x_{1}=1, \\
\frac{\partial}{\partial n} T\left(x_{1}, x_{2}\right) & =\bar{q}, \quad\left(x_{1}, x_{2}\right) \in \Gamma, x_{2}=1 \text { or } x_{1}=0,
\end{aligned}
$$

where $\bar{T}$ and $\bar{q}$ are known functions obtained from the exact solution:

$$
T\left(x_{1}, x_{2}\right)=\left(x_{1}-x_{1}^{2}\right)\left(x_{2}-x_{2}^{2}\right) .
$$

When 40 regular nodes are used (as shown in Figure 8), Figure 9 shows the exact and numerical solutions on the lines $x_{1}=0.5$ and $x_{1}=0.9$. From this figure, it can be seen that the numerical solutions are very consistent with the analytical results, which also shows that the solution of the IBEFM has good accuracy.

In Figure 10, the absolute errors of the numerical solutions obtained by BEFM and IBEFM on the line $x_{1}=0.9$ are given. It can be seen that the solution of the IBEFM still has good accuracy, which also shows the superiority of the IBEFM. The MATLAB programs of the BEFM and IBEFM are repeatedly run 100 times, and their corresponding average CPU time is $3.0335 \mathrm{~s}$ and $3.0170 \mathrm{~s}$, which also shows that the IBEFM has high computational efficiency.

In order to study the convergence of the IFEFM with respect to the radius $\rho$, when the boundary nodes distributions of $16,24,40$, and 60 nodes are, respectively, used, the corresponding absolute errors for different influence 


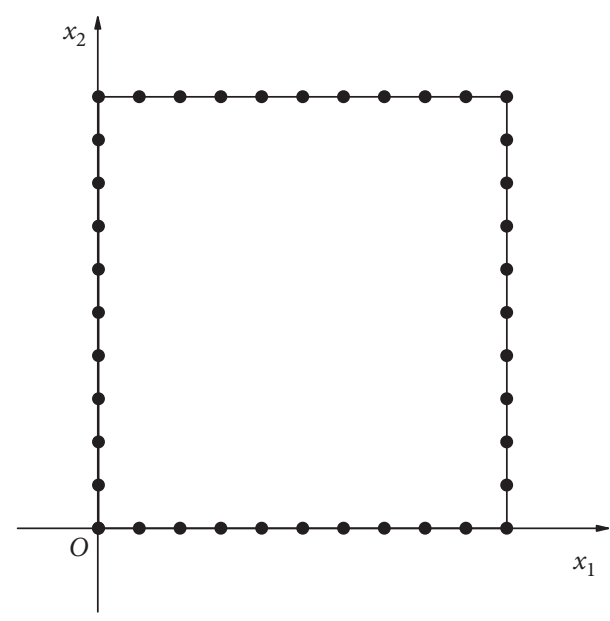

Figure 8: Nodes distribution for Example 2.

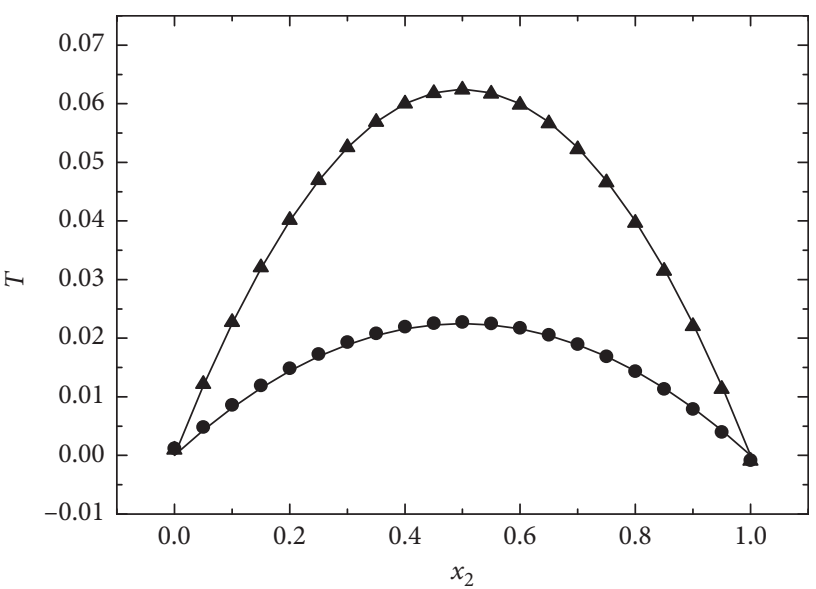

Numerical:
- $x_{1}=0.5$
- Analytical
- $x_{1}=0.9$

Figure 9: The exact and numerical solutions on the lines $x_{1}=0.5$ and $x_{1}=0.9$.

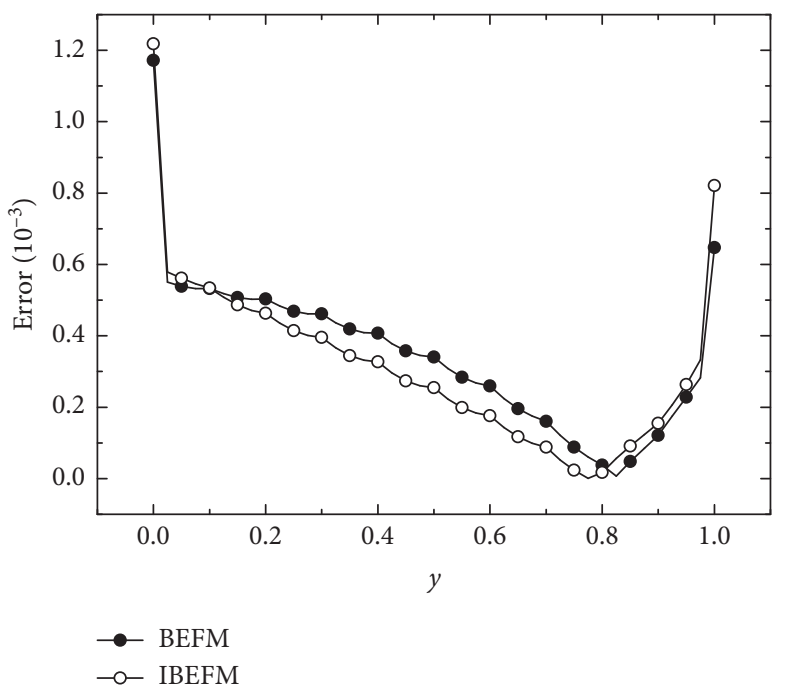

FIgURE 10: The absolute errors of the numerical solutions obtained by the BEFM and IBEFM on the line $x_{1}=0.9$.

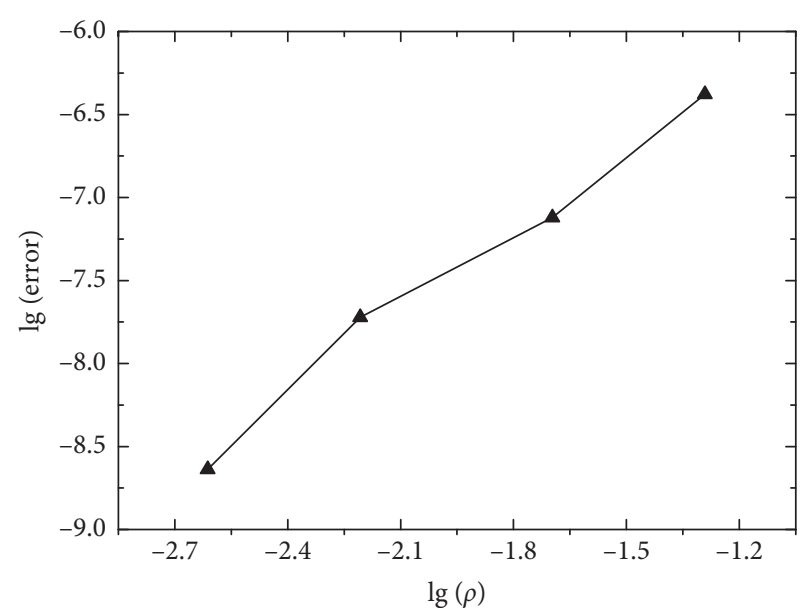

FIgURE 11: The absolute errors for different influence radii at the interior point $(0.4,0.7)$.

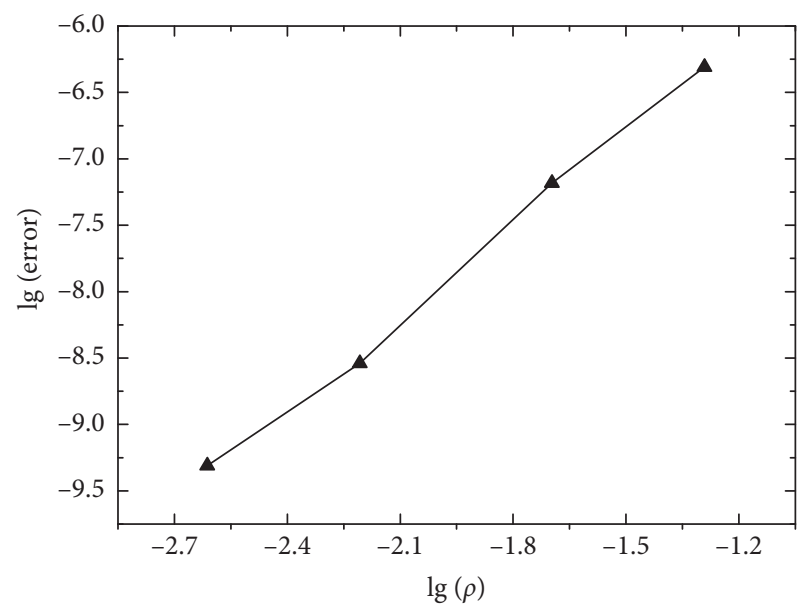

FIgURE 12: The absolute errors for different influence domain radii at the point $(0.5,0.1)$.

domain radii at the interior point $(0.4,0.7)$ and $(0.5,0.1)$ are given in Figures 11 and 12, respectively. It can be seen from these figures that the solution of the IBEFM has a good convergence order, which is consistent with the theoretical results.

\section{Conclusions}

The IBEFM has both the advantages of the high accuracy of the boundary element method and meshless method, and it can also directly apply the boundary conditions. Based on the error estimation of the IMLS method and related matrix theory, this paper studies the error estimation of the IBEFM for two-dimensional potential problems. The relationship between the error and the influence radius and the condition number of the coefficient matrix is studied. The theoretical results show that the upper error limit of the IBEFM solution will decrease with the decrease in the influence domain radius of the boundary nodes and rise with the increase in the condition number of the coefficient matrix. Two numerical examples are provided. The numerical results are in 
good agreement with the theoretical results in this paper, which verifies the correctness of the theoretical results in the article.

\section{Data Availability}

The data used to support the findings of this study are available within the article.

\section{Conflicts of Interest}

The authors declare that there are no conflicts of interest regarding the publication of this paper.

\section{Acknowledgments}

This work was supported by the National Natural Science Foundation of China (Grant no. 11702153), the Natural Science Foundation of Zhejiang Province, China (Grant nos. LY20A010021 and LY20G030025), the Natural Science Foundation of Ningbo City, China (Grant nos. 2018A610196, 2019A610095, and 2019A610048), and National Social Science Foundation of China (18BGL184).

\section{References}

[1] J. Wang and F. Sun, "An interpolating meshless method for the numerical simulation of the time-fractional diffusion equations with error estimates," Engineering Computations, vol. 37, no. 2, pp. 730-752, 2019.

[2] H. Cheng, M. J. Peng, and Y. M. Cheng, "A hybrid improved complex variable element-free Galerkin method for threedimensional potential problems," Engineering Analysis with Boundary Elements, vol. 84, pp. 52-62, 2017.

[3] Z. Lin, F. Liu, D. Wang, and Y. Gu, "Reproducing kernel particle method for two-dimensional time-space fractional diffusion equations in irregular domains," Engineering Analysis with Boundary Elements, vol. 97, pp. 131-143, 2018.

[4] G. R. Liu, "An overview on meshfree methods: for computational solid mechanics," International Journal of Computational Methods, vol. 13, no. 5, Article ID 1630001, 2016.

[5] R. Huang, S. Zheng, Z. Liu, and T. Y. Ng, "Recent advances of the constitutive models of smart materials-hydrogels and shape memory polymers," International Journal of Applied Mechanics, vol. 12, no. 2, Article ID 2050014, 2020.

[6] M. S. Shadloo, G. Oger, and D. Le Touzé, "Smoothed particle hydrodynamics method for fluid flows, towards industrial applications: motivations, current state, and challenges," Computers \& Fluids, vol. 136, pp. 11-34, 2016.

[7] A.-M. Zhang, P.-N. Sun, F.-R. Ming, and A. Colagrossi, "Smoothed particle hydrodynamics and its applications in fluid-structure interactions," Journal of Hydrodynamics, vol. 29, no. 2, pp. 187-216, 2017.

[8] Q. Wu, P. Peng, and Y. Cheng, "The interpolating elementfree Galerkin method for elastic large deformation problems," Science China Technological Sciences, vol. 63, 2020.

[9] J. Lin, C. Zhang, L. L. Sun, and J. Lu, "Simulation of seismic wave scattering by embedded cavities in an elastic half-plane using the novel singular boundary method," Advances in Applied Mathematics and Mechanics, vol. 10, no. 2, pp. 322342, 2018.

[10] B. Fornberg and N. Flyer, "Solving PDEs with radial basis functions," Acta Numerica, vol. 24, pp. 215-258, 2015.
[11] J. Lin, S. Y. Reutskiy, and J. Lu, "A novel meshless method for fully nonlinear advection-diffusion-reaction problems to model transfer in anisotropic media," Applied Mathematics and Computation, vol. 339, pp. 459-476, 2018.

[12] Z.-J. Fu, Q. Xi, W. Chen, and A. H.-D. Cheng, "A boundarytype meshless solver for transient heat conduction analysis of slender functionally graded materials with exponential variations," Computers \& Mathematics with Applications, vol. 76, no. 4, pp. 760-773, 2018.

[13] E. Shivanian, "Meshless local Petrov-Galerkin (MLPG) method for three-dimensional nonlinear wave equations via moving least squares approximation," Engineering Analysis with Boundary Elements, vol. 50, pp. 249-257, 2015.

[14] Y. Deng, C. Liu, M. Peng, and Y. Cheng, "The interpolating complex variable element-free Galerkin method for temperature field problems," International Journal of Applied Mechanics, vol. 7, no. 2, Article ID 1550017, 2015.

[15] H. Cheng, M. Peng, Y. Cheng, and Z. Meng, "The hybrid complex variable element-free Galerkin method for 3D elasticity problems," Engineering Structures, vol. 219, Article ID 110835, 2020.

[16] Q. Liu, F. Liu, Y. T. Gu, P. Zhuang, J. Chen, and I. Turner, “A meshless method based on point interpolation method (PIM) for the space fractional diffusion equation," Applied Mathematics and Computation, vol. 256, pp. 930-938, 2015.

[17] Y. Gu, C.-M. Fan, and R.-P. Xu, "Localized method of fundamental solutions for large-scale modeling of two-dimensional elasticity problems," Applied Mathematics Letters, vol. 93, pp. 8-14, 2019.

[18] H. Cheng, M. J. Peng, and Y. M. Cheng, "The dimension splitting and improved complex variable element-free Galerkin method for 3-dimensional transient heat conduction problems," International Journal for Numerical Methods in Engineering, vol. 114, no. 3, pp. 321-345, 2018.

[19] J. Cheng, "Analyzing the factors influencing the choice of the government on leasing different types of land uses: evidence from Shanghai of China," Land Use Policy, vol. 90, Article ID 104303, 2020.

[20] J. Cheng, "Data analysis of the factors influencing the industrial land leasing in Shanghai based on mathematical models," Mathematical Problems in Engineering, vol. 2020, Article ID 9346863, 11 pages, 2020.

[21] P. Lancaster and K. Salkauskas, "Surfaces generated by moving least squares methods," Mathematics of Computation, vol. 37, no. 155, p. 141, 1981.

[22] M. Dehghan and M. Abbaszadeh, "Proper orthogonal decomposition variational multiscale element free Galerkin (POD-VMEFG) meshless method for solving incompressible Navier-Stokes equation," Computer Methods in Applied Mechanics and Engineering, vol. 311, pp. 856-888, 2016.

[23] S. Wu and Y. Xiang, "A coupled interpolating meshfree method for computing sound radiation in infinite domain," International Journal for Numerical Methods in Engineering, vol. 113, no. 9, pp. 1466-1487, 2018.

[24] S. Chen, W. Wang, and X. Zhao, "An interpolating elementfree Galerkin scaled boundary method applied to structural dynamic analysis," Applied Mathematical Modelling, vol. 75, pp. 494-505, 2019.

[25] J. Wang and F. Sun, "A hybrid variational multiscale elementfree Galerkin method for convection-diffusion problems," International Journal of Applied Mechanics, vol. 11, no. 7, Article ID 1950063, 2019.

[26] J. Wang and F. Sun, "A hybrid generalized interpolated element-free Galerkin method for Stokes problems," 
Engineering Analysis with Boundary Elements, vol. 111, pp. 88-100, 2020.

[27] E. Shivanian, "A new spectral meshless radial point interpolation (SMRPI) method: a well-behaved alternative to the meshless weak forms," Engineering Analysis with Boundary Elements, vol. 54, pp. 1-12, 2015.

[28] J. F. Wang, F. X. Sun, Y. M. Cheng, and A. X. Huang, "Error estimates for the interpolating moving least-squares method," Applied Mathematics and Computation, vol. 245, pp. 321-342, 2014.

[29] Y. Cheng, F. Bai, C. Liu, and M. Peng, "Analyzing nonlinear large deformation with an improved element-free Galerkin method via the interpolating moving least-squares method," International Journal of Computational Materials Science and Engineering, vol. 5, no. 4, Article ID 1650023, 2016.

[30] M. Dehghan and M. Abbaszadeh, "Interpolating stabilized moving least squares (MLS) approximation for 2D elliptic interface problems," Computer Methods in Applied Mechanics and Engineering, vol. 328, pp. 775-803, 2018.

[31] Y. M. Cheng, F. N. Bai, and M. J. Peng, "A novel interpolating element-free Galerkin (IEFG) method for two-dimensional elastoplasticity," Applied Mathematical Modelling, vol. 38, no. 21-22, pp. 5187-5197, 2014.

[32] T. Zhang and X. Li, "Meshless analysis of Darcy flow with a variational multiscale interpolating element-free Galerkin method," Engineering Analysis with Boundary Elements, vol. 100, pp. 237-245, 2019.

[33] R. Singh and K. M. Singh, "Interpolating meshless local Petrov-Galerkin method for steady state heat conduction problem," Engineering Analysis with Boundary Elements, vol. 101, pp. 56-66, 2019.

[34] Y. Cheng and M. Peng, "Boundary element-free method for elastodynamics," Science in China Series G, vol. 48, no. 6, pp. 641-657, 2005.

[35] K. M. Liew and Y. Cheng, "Complex variable boundary element-free method for two-dimensional elastodynamic problems," Computer Methods in Applied Mechanics and Engineering, vol. 198, no. 49-52, pp. 3925-3933, 2009.

[36] M. Peng and Y. Cheng, "A boundary element-free method (BEFM) for two-dimensional potential problems," Engineering Analysis with Boundary Elements, vol. 33, no. 1, pp. 77-82, 2009.

[37] L. Chen, X. Liu, and X. Li, "The boundary element-free method for 2D interior and exterior Helmholtz problems," Computers \& Mathematics with Applications, vol. 77, no. 3, pp. 846-864, 2019.

[38] X. Li, S. Zhang, Y. Wang, and H. Chen, "A complex variable boundary element-free method for potential and helmholtz problems in three dimensions," International Journal of Computational Methods, vol. 17, no. 2, Article ID 1850129, 2020.

[39] H. Ren, Y. Cheng, and W. Zhang, "An interpolating boundary element-free method (IBEFM) for elasticity problems," Science China Physics, Mechanics and Astronomy, vol. 53, no. 4, pp. 758-766, 2010.

[40] H. Ren and Y. Cheng, "The interpolating element-free Galerkin (IEFG) method for two-dimensional elasticity problems," International Journal of Applied Mechanics, vol. 3, no. 4, pp. 735-758, 2011.

[41] X. Li, "An interpolating boundary element-free method for three-dimensional potential problems," Applied Mathematical Modelling, vol. 39, no. 10-11, pp. 3116-3134, 2015.

[42] J. Wang, J. Wang, F. Sun, and Y. Cheng, "An interpolating boundary element-free method with nonsingular weight function for two-dimensional potential problems," International Journal of Computational Methods, vol. 10, no. 6, Article ID 1350043, 2013.

[43] Q. Wang, W. Zhou, Y. T. Feng, G. Ma, Y. Cheng, and $\mathrm{X}$. Chang, "An adaptive orthogonal improved interpolating moving least-square method and a new boundary elementfree method," Applied Mathematics and Computation, vol. 353, pp. 347-370, 2019.

[44] F. X. Sun, J. F. Wang, Y. M. Cheng, and A. X. Huang, "Error estimates for the interpolating moving least-squares method in $n$-dimensional space," Applied Numerical Mathematics, vol. 98, pp. 79-105, 2015.

[45] J. F. Wang, S. Y. Hao, and Y. M. Cheng, "The error estimates of the interpolating element-free Galerkin method for twopoint boundary value problems," Mathematical Problems in Engineering, vol. 2014, Article ID 641592, 12 pages, 2014. 